\title{
Real-time Automatic Deceit Detection from Involuntary Facial Expressions
}

\author{
Zhi Zhang ${ }^{\dagger}$, Vartika Singh ${ }^{\dagger}$, Thomas E. Slowe ${ }^{\ddagger}$, Sergey Tulyakov ${ }^{\dagger}$, and Venugopal Govindaraju ${ }^{\dagger}$ \\ ${ }^{\dagger}$ Center for Unified Biometrics and Sensors \\ University at Buffalo, NY, USA \\ \{zhizhang, vsingh2, tulyakov, venu\}@cubs.buffalo.edu \\ $\ddagger$ CUBRC, Buffalo, NY, USA \\ slowe@cubrc.org
}

\begin{abstract}
Being the most broadly used tool for deceit measurement, the polygraph is a limited method as it suffers from human operator subjectivity and the fact that target subjects are aware of the measurement, which invites the opportunity to alter their behavior or plan counter-measures in advance. The approach presented in this paper attempts to circumvent these problems by unobtrusively and automatically measuring several prior identified Deceit Indicators (DIs) based upon involuntary, so-called reliable facial expressions through computer vision analysis of image sequences in real time. Reliable expressions are expressions said by the psychology community to be impossible for a significant percentage of the population to convincingly simulate, without feeling a true inner felt emotion. The strategy is to detect the difference between those expressions which arise from internal emotion, implying verity, and those expressions which are simulated, implying deceit. First, a group of Facial Action Units (AUs) related to the reliable expressions are detected based on distance and texture based features. The DIs then can be measured and finally a decision of deceit or verity will be made accordingly. The performance of this proposed approach is evaluated by its real time implementation for deceit detection.
\end{abstract}

\section{Introduction}

The task of estimating credibility of others is engaged by people daily during normal social circumstances. Although practiced daily, research in psychology has shown that most people are bad at judging deceit or verity in others. An automatic system that could aid humans in this judgment would be highly valuable. As one of the most widely utilized systems, polygraph testing is proved to be reliable for this cause, however, it follows an overt approach. A covert system could leverage the fact that the subject would be un- aware that they are being scrutinized by a reliable deceit detector.

Law enforcement and private investigators rely heavily upon the testimony of witnesses and suspects to solve criminal cases. Courts are centered upon the assumption that witnesses are credible. Agents from border patrol, airlines, and other government and corporate officials require accurate and truthful information from subjects in order to maintain proper safety for the benefit of society. Based upon analysis of face, voice and body behavior, training aids for humans to detect deceit have been developed in the psychology community. While these forms of training do increase humans' ability to detect deceit, humans are subjective and qualitative in nature.

Several research efforts are underway to replace human with an automatic system for deceit detection. In [2], thermal video is employed to generate an estimate of deceit. While a promising area of research, it requires thermal video equipment, which is expensive and could potentially limit broad deployment. The work seen in [12] and other efforts leveraging similar modalities, require active sensing, which could possibly be detected and counter-measured by the subject. Facial Action Coding System (FACS) is a framework created by Ekman et al. [7] for the study and classification of facial expressions, and it was widely utilized in facial expression analysis and other related research fields. In FACS, expressions are represented as combinations of fundamental facial Action Units (AUs) based upon the underlying musculature of the face.

During the research in psychology of facial behavior, Ekman et al. [8] found that while culture and society affect voluntary use of facial expression, there exist expressions that arise as a result of inner felt emotion, and are thus involuntary. Naturally, these facial behaviors transcend culture, sex, and age. It was postulated that if voluntarily arising expressions are visibly different from those expressions arising from involuntary triggers, a contradiction can be detected, and deceit can be inferred. 


\begin{tabular}{|l|c|}
\hline Expressions & AU Combinations \\
\hline \hline Anger & AU 23 \\
Enjoyment & AU $6+12$ \\
Fear & AU $1+2+4$ or AU 20 \\
Sadness & AU $1+4+15$ \\
\hline
\end{tabular}

Table 1. Four reliable expressions with their associated facial AUs.

In [6], based upon their experience in facial behavior psychology, Ekman proposed several deceit indicators brought on by these involuntary movements of the face. A few specific expressions were postulated to be useful when searching for evidence of suppressed emotions, which would imply deceit when identified. These expressions rely upon 'reliable muscles', which cannot be voluntarily controlled by the vast majority of people [6].

Table 1 shows the four expressions and their specific combinations of AUs that, according to Ekman's belief, cannot be triggered involuntarily by majority of people. In the case of enjoyment, for example, there is a measurable difference between a genuine smile (coined Duchenne Smile) and a fake or 'polite' one (coined Non-Duchenne Smile). Duchenne smiles stimulate both the zygomatic major muscle (AU 12) and the orbicularis oculi muscle (AU 6), while Non-Duchenne smiles only stimulate the zygomatic major muscle. The orbicularis oculi muscles that encircle each eye aid in restricting and controlling the skin around the eyes, and they cannot be moved into the correct smile position voluntarily by most of the population. Only a natural feeling of happiness or enjoyment can move these muscles into proper happiness position. Table 3 shows the details of AU 6, AU 12 and other AUs related to reliable expressions.

Several research groups $[1,5,13,14]$ have undertaken research to detect the most common facial AUs and their combinations defined by Ekman [7]. These systems are not tuned for specific application, and it is unclear how their systems would perform when tasked with solving the deceit and verity classification problem. Burgoon et al. [3] proposed an approach that measures deceit and verity from the whole body behavior, where body actions or gestures are measured overtime to produce an estimate of credibility.

The work of Slowe et al. [11] established credibility to the area of measuring deceit indication from facial behaviors. Through manual tests and basic algorithm implementations, a group of deceit indicators (DIs) were assessed on verified video data acquired by CUBRC and the Center for Unified Biometrics and Sensors (CUBS). Table 2 shows their results, which leveraged the feasibility of an automatic system based on reliable expressions that could detect deceit through those DIs.

We have used Gabor Wavelets based feature extraction which is insensitive to illumination variety, individual dif-

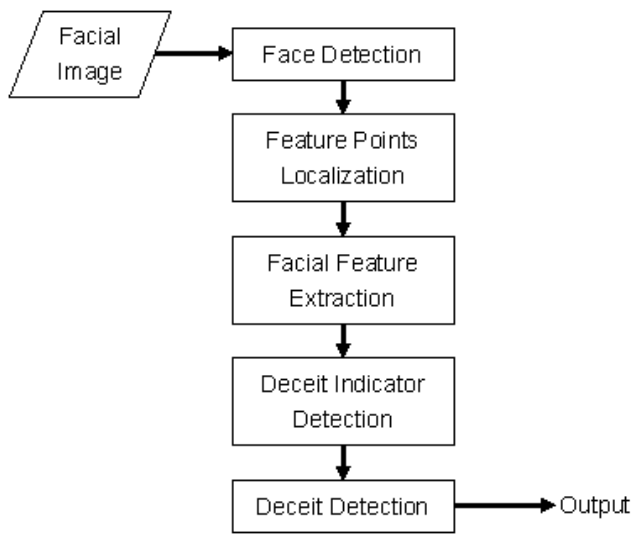

Figure 1. The framework of real-time deceit detection system based on reliable facial expressions through DI detections.

ference and small scale changes in filter properties [10] [9].

The approach described in this paper is based on visible facial behaviors indicated as implying deceit by the psychology community. A new approach for automatic deceit detection based on reliable facial expressions through detecting deceit indicators. In this approach, AUs related to the four reliable expressions are decomposed into major components (MCs), and each of MCs is detected based on either distance based features or texture based features or both. Deceit indicators (DIs) will be detected based on the result of MC detections. Finally, a decision of deceit or verity will be made based on the result of DI detections. Figure 1 outlined this system, in which the first two modules are done manually. The organization of this paper is as follows: In Section 2, we will overview the definitions and detections of DIs for the four reliable expressions. The datasets are described in Section 3. Experiment setups, results and evaluations will be given in Section 4. Finally the concluding remarks will be given in Section 5.

\section{Deceit Detection in the Face}

Major Components (MCs) of AUs are introduced by interpretations of the AU definitions given in [7] for better understanding and facilitation of $\mathrm{AU}$ detections. For this project, seven specific AUs (AU 1, 2, 4, 6, 12, 15, and 23) cover our interest, and the FACS definitions and the interpretations in MCs of them are shown in Table 3.

Due to the nature of the MCs, two types of features are applied in this approach: distance based features (DBF) and texture based features (TBF). Among the 16 defined MCs, nine (MC 2, 3, 4, 5, 9, 12, 14, 15, and 16) can be represented with DBFs and the rest can be represented with TBFs. In order to process these features, 58 facial points are defined, as shown in Figure 2, and manually labeled for all the images in the CUBRC-CEDAR dataset. The definitions and extrac- 


\begin{tabular}{|l|c|c|c|c|}
\hline & Anger & Enjoyment & Fear & Sadness \\
\hline \hline Defined DIs & AU 23 & AU 6+12 & AU 2 & AU 15 \\
\hline DI Presenting Rate in Verity & $89.5 \%$ & $98.4 \%$ & $80.0 \%$ & $97.7 \%$ \\
\hline DI Presenting Rate in Deceit & $2.7 \%$ & $20.5 \%$ & $33.3 \%$ & $0.0 \%$ \\
\hline
\end{tabular}

Table 2. Manual test results of correlation between specific DI and instances of deceit/verity by Slowe et al. [11].

\begin{tabular}{|l|l|l|}
\hline AU & FACS Definition & Major Components \\
\hline \hline AU 1 & Inner brow raiser & 1. Horizontal forehead wrinkle. \\
& & 2. Inner brows raiser. \\
\hline AU 2 & Outer brow raiser & 3. Outer brows raiser. \\
\hline AU 4 & Brows lower & 4. Brows lower. \\
& & 5. Inner brows raiser. \\
& & 6. Vertical wrinkles between brows. \\
& & 7. Horizontal wrinkles at root of nose. \\
\hline AU 6 & Cheek raiser and lid compressor & 8. Cheek raiser. \\
& & 9. Eye aperture narrower. \\
& & 10. Lower eyelid furrow deepen. \\
& & 11. Crow's Feet wrinkle. \\
\hline AU 12 & Lip corner puller & 12. Corners of lips up. \\
& & 13. Nasolabial furrow deepen. \\
\hline AU 15 & Lip corner depressor & 14. Corners of lips down. \\
& & 15. Lower lip stretched horizontally. \\
\hline AU 23 & Lip tightener & 16. Red parts of lips narrowed. \\
\hline
\end{tabular}

Table 3. FACS Definitions of AUs of interest and their representations in major components.

tions of the features would be introduced in details in the following subsections.

The nine DBF related MCs are mainly concentrated on two regions, eye region and mouth region. In this work, Euclidean distance is applied when calculating distance between two facial points or distance between one facial point to a certain line formed by the other two facial points. Relative distances are introduced to overcome the problem of various sized faces, where distance between two inner corners of eyes is used as reference distance, or $R D$. In the rest of the paper, distance refers to relative Euclidean distance, if not specified.

For the TBF related MCs, the images are first scaled and grayscale normalized to minimize the in between difference between them. There are in all 7 TBF based MCs. But, due to lack of data, MC 8 has not been studied/used here. The approach involves obtaining the Gabor wavelets at selected points in the face, where maximum possible variation is found due to muscular involvement during facial expressions. They are calculated over a local neighborhood of the point selected for a particular MC. From these transforms, four moments are calculated for each point transform. The wavelets are calculated for different orientations and frequencies. These orientations and frequencies are optimized based on the prior knowledge of the nature of furrows/wrinkles/creases in the particular point where the wavelets are being calculated. The classification for all the MCs is binary, based on their presence or absence. The classification is done using SVM with RBF kernel. We used LIBSVM [4] with default parameters.

\subsection{Anger}

In attempt to fake anger facially, people would perform multiple combined expressions that are meant to trick others into believing that they are angry. Nonetheless, it is rare that a person will actually produce AU 23 without being truly angry. Naturally, the anger DI is characterized by AU 23.

In our 58 facial points definitions, three points are defined on each of the lips. In order to detect AU 23 or MC 16 , three distances between feature points located on the upper and lower lips are calculated.

\subsection{Enjoyment}

In order to detect deceit in enjoyment, the combination of AU 6 and AU 12 is applied, as genuine smile or Duchenne smile should display both these AUs while fake smile or Non-Duchenne smile only displays AU 12. Thus, independent detections of these two AUs are required for this project.

In order to detect AU6, MC 8-11 are defined, among 
which MC 9 is represented by DBFs. Three facial points are defined along each of the four eyelids. Six distances are calculated among those points to represent MC 9. Due to their natures, MC 8, 10 and 11 are represented by TBFs. Texture analysis is performed on two corresponding facial regions for each of these MCs. MC 12 and 13 are defined to detect AU 12. Eight facial points are defined around mouth region. Six distances are calculated among those points to represent MC 12. For MC 13, texture of two facial regions besides nose are analyzed, and Gabor wavelet features are extracted.

\subsection{Fear}

As shown in Table 1, two different expressions, AU combination $1+2+4$ and $\mathrm{AU} 20$, can be applied as DI for fear. Due to the lack of instances of AU 20 in our current CUBRC-CUBS dataset, only AU combination 1+2+4 is considered.

In order to detect AU 1, MC 1 and MC 2 are defined. For MC 1, texture analysis of the forehead region is performed, and Gabor wavelet features are extracted. For MC 2 , two distances between two inner eyebrow tips and the line formed by the two inner eye corners are calculated. Similarly, two distances between two outer eyebrow tips and the line formed by the two inner eye corners are calculated to detect AU 2 or MC 3. In order to detect AU 4, MC 4-7 are defined. Three distances for each eyebrow are calculated, and six distances in total to represent MC 4. One distance between two inner eyebrow tips are calculated to represent MC 5. Texture of the region between two eyebrows is analyzed and Gabor wavelet features are extracted to represent MC 6. Texture analysis of the region at the root of nose is performed and Gabor wavelet features are extracted to represent MC 7.

\subsection{Sadness}

The sadness DI is represented by combination of AU 1, AU 4 and AU15 with AU 15 being the critical indicator of deceit detection for sadness expressions according to researchers in the academic psychology community.

In order to detect $\mathrm{AU}$ 15, MC 14 and 15 are defined. Similar to MC 14, MC 15 is also represented by the same six distances among the facial points on mouth region. Distance between the two outer corners of mouth is calculated to represent MC 15.

\section{Dataset Acquisitions and Preprocessing}

344 facial images of 12 individuals, 6 males and 6 females, are extracted from the verified video data acquired by CUBRC and the Center for Unified Biometrics and Sensors (CUBS) for training and testing the system. About $2 / 3$ of these images show genuine expressions, and the rest show
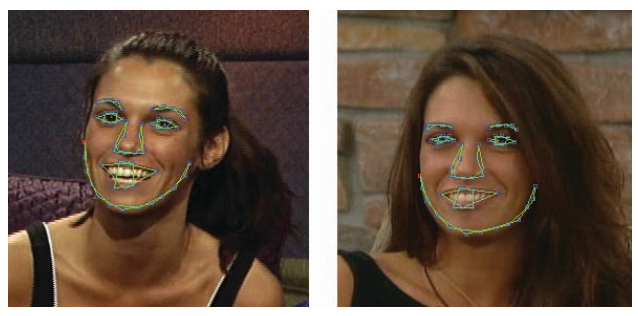

Figure 2. The genuine, the left, and fake smiles of one subject in the available dataset. Notice that AU 6 is absent in the fake smile. Orignal images are copyrighted to Endemol USA Inc.

deceitful expressions. Figure 2 shows two sample images from the dataset showing genuine and fake smiles of the same subject. For all these images, 58 facial points are manually labelled and ground truth of deceit/verity, AUs and MCs are manually input.

\section{Experiment Results and Discussion}

\subsection{Experiment Results of DBFs}

Due to the fact that only limited number of samples are available in the dataset, statistic analysis was conducted on all the samples to verify that the distributions of the distance based features are different and hence separable for the two classes, namely presence or absence of certain MC. Wilcoxon rank sum test was applied for this purpose, and according to the results of this test, all of the features, except two features for MC 4, have $\mathrm{p}$ values lower than $5 \%$. These results confirmed that most of the distance based features can be applied to the task of detecting the presence of certain DBF represented MC.

In this part of experiment, half of all the available samples are selected randomly as training samples and the rest are used for testing purpose. Simple thresholding was applied to the MCs that are represented by small number of DBFs, while supporting vector machines (SVM) are trained for MCs that are represented by more than 3 DBFs.

Table 4 shows the detecting results of MCs that are represented by DBFs. Among these results, MC 15 and MC 4 have the lowest accuracy. MC 15, being one of the weak MC for AU 15 and represented by distance between two outer corners of mouth, isn't reliable due to the fact that for AUs other than AU 15, this distance may also be big, e.g. when mouth is open wide. The reason for MC 4 being unreliable could be the fact that different people have different distance between eyebrows and eyes. In fact, the results of Wilcoxon rank sum test on these two MCs indicate that the DBFs represent them have larger $\mathrm{p}$ values than those of the rest of the MCs.

One way to improve the performance of these DBFs is to substitute these distances of a certain subject in a given ex- 


\begin{tabular}{|c|c|c|}
\hline MC & $\begin{array}{c}\text { No. of } \\
\text { Features }\end{array}$ & Accuracy \\
\hline \hline MC 2 & 2 & $68.67 \%$ \\
\hline MC 3 & 2 & $74.07 \%$ \\
\hline MC 4 & 6 & $63.79 \%$ \\
\hline MC 5 & 1 & $77.71 \%$ \\
\hline MC 9 & 6 & $68.51 \%$ \\
\hline MC 12 & 6 & $78.60 \%$ \\
\hline MC 14 & 6 & $77.05 \%$ \\
\hline MC 15 & 1 & $53.01 \%$ \\
\hline MC 16 & 3 & $71.44 \%$ \\
\hline
\end{tabular}

Table 4. Test results of detecting MCs represented by DBFs.

\begin{tabular}{|c|c|}
\hline MC & Accuracy \\
\hline \hline MC 1 & $78.33 \%$ \\
\hline MC 6 & $82.50 \%$ \\
\hline MC 7 & $92.86 \%$ \\
\hline MC 10 & $71.15 \%$ \\
\hline MC 11 & $88.68 \%$ \\
\hline MC 13 & $97.18 \%$ \\
\hline
\end{tabular}

Table 5. Test results of detecting MCs represented by TBFs.

pression for the difference between these distances and the same set of distances from this subject in a neutral expression. Restricted to the available dataset, this approach has not been implemented.

\subsection{Experiment Results of TBFs}

The results obtained from the classification of TBFs, for each MC, varied a lot depending on the size of the data set used, but over all they are quite optimistic. Due to small data set being used, even a difference of single data in testing sets causes a huge variation in the output accuracies. All the classifications were done using LIBSVM library, with Radial basis fucntions as kernels and default parameters. The data was scaled before the classification. Table 5 shows the results of detecting results of MCs that are represented by TBFs.

\subsection{Experiment Results of Deceit Indicators}

The focus of this work is to establish credibility to the area of measuring deceit indication from involuntary facial expressions, therefore the experiments for deceit indicators were conducted separately for these four expressions, namely anger, enjoy, fear and sadness. For each expression type, samples of both deceit and verity in the CUBRCCEDAR dataset were distributed randomly into the training and testing data sets. Distance based features and texture based features for each of these expression were applied, and their results are combined. Presently they are being

\begin{tabular}{|l|c|c|c|c|}
\hline Expression & Anger & Enjoyment & Fear & Sadness \\
\hline \hline Accuracy & $86.02 \%$ & $73.16 \%$ & $80.46 \%$ & $90.15 \%$ \\
\hline
\end{tabular}

Table 6. Test results of detecting deceit from involuntary facial expressions.

combined based on simple averaging.

Table 6 shows the testing results of detecting deceit from involuntary facial expressions based on combination of distance based features and texture based features.

\section{Conclusion and Future Work}

In this work, an automatic system that could measure deceit indications from facial behaviors was proposed and implemented. The authors propose to advance the work by trying to get better results with less data. The system is currently built for static data. We propose to further the work for real time systems by enhancing the speed of the feature extraction methods and their further combination to predict deceit or verity.

\section{References}

[1] M. S. Bartlett, G. C. Littlewort, M. G. Frank, C. Lainscsek, I. Fasel, and J. R. Movellan. Automatic recognition of facial actions in spontaneous expressions. Journal of Multimedia, 1(6):22-35, 2006.

[2] P. Buddharaju, J. Dowdall, P. Tsiamyrtzis, D. Shastri, I. Pavlidis, and M. G. Frank. Automatic THErmal Monitoring System (ATHEMOS) for Deception Detection. In IEEE Conf. on Computer Vision and Pattern Recognition, San Diego, CA, USA, 2005.

[3] J. Burgoon, M. Adkins, J. Kruse, M. L. Jensen, T. Meservy, S. Lu, G. Tsechpenakis, and D. Metaxas. An approach for intent identification by building on deception detection. In Int. Conf. on System Sciences, Big Island, Hawaii, USA, January 2005.

[4] C. Chang and C. Lin. LIBSVM: a library for support vector machines, 2001. Software available at http://www.csie.ntu.edu.tw/ cjlin/libsvm.

[5] J. F. Cohn. Foundations of human computing: Facial expression and emotion. In 8th Int. Conf. on Multimodal Interfaces (ICMI'06), Banff, Canada, November 2006.

[6] P. Ekman. Telling Lies: Clues to Deceit in the Marketplace, Politics, and Marriage. W. W. Norton, New York, USA, 1992.

[7] P. Ekman, W. V. Friesen, and J. C. Hager. The Facial Action Coding System. Research Nexus eBook, Salt Lake City, UT, USA, 2002.

[8] P. Ekman, E. R. Sorenson, and W. V. Friesen. Pancultural elements in facial displays of emotions. Science, 164(3875):8688, 1969.

[9] S. E. Grigorescu, N. Petkov, and P. Kruizinga. Comparison of texture features based on gabor filters. Image Processing, IEEE trans., 11(10), October 2002. 
[10] P. Moreno, A. Bernardino, and J. Santos-Victor. Gabor parameter selection for local feature detection. In 2nd Iberian Conf. on Pattern Recognition and Image Analysis, Estoril, Portugal, June 2005.

[11] T. E. Slowe and V. Govindaraju. Automatic deceit indication through reliable facial expressions. In 5th IEEE Workshop on Automatic Identification Advanced Technologies (AutoID 2007), Alghero, Italy, June 2007.

[12] E. M. Staderini. An UWB radar based stealthy 'Lie Detector'. Online technical report.

[13] Y. Tian, T. Kanade, and J. Cohn. Recognizing action units for facial expression analysis. Technical Report CMU-RITR-99-40, Robotics Institute, Carnegie Mellon University, December 1999.

[14] Y. Zhang and Q. Ji. Active and dynamic information fusion for facial expression understanding from image sequences. IEEE Trans. Pattern Anal. Mach. Intell., 27(5), May 2005. 